Draft version DeCEmber 14, 2020

Typeset using $\mathrm{LAT}_{\mathrm{E}} \mathrm{X}$ preprint2 style in AASTeX63

\title{
Departure from the Exact Location of Mean Motion Resonances Induced by the Gas Disk in the Systems Observed by Kepler
}

\author{
Su Wang, ${ }^{1}$ D.N.C. Lin,${ }^{2,3}$ Xiaochen Zheng, ${ }^{4}$ And Jianghui Ji ${ }^{1,5}$ \\ ${ }^{1}$ CAS Key Laboratory of Planetary Sciences, Purple Mountain Observatory, \\ Chinese Academy of Sciences, Nanjing 210008, China \\ ${ }^{2}$ Department of Astronomy and Astrophysics, University of California, \\ Santa Cruz, CA 95064, USA \\ ${ }^{3}$ Institute for Advanced Studies, Tsinghua University, Beijing 100086, China \\ ${ }^{4}$ Department of Astronomy, Tsinghua University, Beijing 100086, China \\ ${ }^{5}$ CAS Center for Excellence in Comparative Planetology, Hefei 230026, China
}

\begin{abstract}
The statistical results of transiting planets show that there are two peaks around 1.5 and 2.0 in the distribution of orbital period ratios. A large number of planet pairs are found near the exact location of mean motion resonances (MMRs). In this work, we find out that the depletion and structures of gas disk play crucial roles in driving planet pairs out of exact location of MMRs. Under such scenario, planet pairs are trapped into exact MMRs during orbital migration firstly and keep migrating in a same pace. The eccentricities can be excited. Due to the existence of gas disk, eccentricities can be damped leading to the change of orbital period. It will make planet pairs depart from the exact location of MMRs. With depletion timescales larger than $1 \mathrm{Myr}$, near MMRs configurations are formed easily. Planet pairs have higher possibilities to escape from MMRs with higher disk aspect ratio. Additionally, with weaker corotation torque, planet pairs can depart farther from exact location of MMRs. The final location of the innermost planets in systems are directly related to the transition radius from optically thick region to inner optically thin disk. While the transition radius is smaller than 0.2 $\mathrm{AU}$ at the late stage of star evolution process, the innermost planets can reach around 10 days. Our formation scenario is a possible mechanism to explain the formation of near MMRs configuration with the innermost planet farther than $0.1 \mathrm{AU}$.
\end{abstract}

Keywords: planetary systems: planets and satellites: formation: protoplanetary disks.

\section{INTRODUCTION}

The Kepler mission and its follow-up program K2 have released over 6000 planetary candidates, including $\sim 2700$ confirmed planets and more than 3300 candidates yet to be confirmed (Borucki et al. 2010; Batalha et al. 2013; Mazeh et al. 2013; Fabrycky et al. 2014;
Dressing et al. 2017; Kunimoto \& Matthews 2020). Among them, there are hundreds of confirmed multiple planetary systems. The population of the discovered planets provides us a good sample to study the dynamics and formation of planetary systems (Moriarty \& Ballard 2016; Gong \& Ji 2017; Mills \& Fabrycky 2017; 
Wang \& Ji 2017; Gong \& Ji 2018; He et al. 2019; Yang et al. 2020; Zhang 2020). From the statistic results, we find that there are plenty of planet pairs in the configuration of near MMRs especially the first order resonances, such as 2:1 and 3:2 MMRs (Lissauer et al. 2011; Wang et al. 2012; Lee et al. 2013; Wang \& Ji 2014, 2017; Antoniadou \& Libert 2020). However, the peaks in the distribution of period ratios are shown not in the exact location of MMRs, but a little departure from them (Wang et al. 2012; Lee et al. 2013; Steffen et al. 2013; Wang \& Ji 2017; Wu et al. 2019; Pan et al. 2020). Most of the planet pairs pipe up at the location larger than the exact MMRs. Near 3:2 MMR, the maximum fraction of planet pairs can reach $\sim 3 \%$ for the period ratio within an interval of 0.025 , while the maximum fraction near 2:1 MMRs is about $2 \%$ for the period ratio within an interval of 0.025. The Transiting Exoplanet Survey Satellite (Tess) has started operations in July 2018 to detect transiting planets in $85 \%$ of the sky (Ricker et al. 2015). Up to now, there are more than 80 planets have been confirmed and over two thousand planetary candidates to be confirmed. TOI-125 and TOI-270 which are confirmed by TESS are multiple planetary systems bearing planets in near MMRs configurations (Quinn et al. 2019; Nielsen et al. 2020). More systems with similar configurations are expected to be found by TESS mission to enlarge the number of planet pairs in near MMRs.

Several mechanisms are proposed to explain the formation of configuration near exact MMRs. The major scenario of the tidal effect produces near MMRs for the systems with planets very close to the central star (Papaloizou \& Terquem 2010; Lithwick \& Wu 2012; Batygin \& Morbidelli 2013; Delisle et al. 2014). Lee et al. (2013) analyzed the systems with planets less than $2 R_{\oplus}$ in radius and showed that if they are rocky with $Q / k_{2} \sim 100$, planet pairs can form near MMRs configuration because of tidal damping. But for the planets larger than $2 R_{\oplus}$ or with the distance from the central star farther than 0.1 AU where the tidal effect is not strong enough, the formation of near MMRs configuration is not clear.

The magnetospheric cavity with a one-side torque disk plays an alternative role in the formation of near MMRs configurations (Koenigl 1991; Liu et al. 2015, 2017; Liu \& Ji 2020). They explored the effect of various planet masses, disk accretion rates, stellar magnetic field strengths, and depletion timescales of gas disks. The direction of type I migration in the edge of the cavity is outward. The configuration of the planet pairs which can be trapped into MMRs at the very beginning will be rearranged. However, in this scenario, a special gas disk is required and the final period ratio is related to the mass ratio of two planets.

A late orbital instability after disk depletion has been suggested to explain the formation of near MMRs configuration (Izidoro, et al 2017; Ogihara et al. 2018; Lambrechts, et al. 2019). They assumed that planets formed a chain resonance configuration after slow migration with a timescale of about 1 Myr. The orbital instability during the gas depletion phase will make close-in super-Earths forming non-resonant observed configurations. The final period ratio between planets depends on their masses. But they just investigated the influence of planet mass on the final stage. The planet mass growth process (Petrovich et al. 2013) or interaction with planetesimals (Chatterjee \& Ford 2015) can lead to the formation of near MMRs configurations. However, the depletion timescale and structures of gas disks will affect the amplitude that departs from the exact MMRs.

Based on the classical migration model, we present a formation scenario of near MMRs configuration. First of all, planets are formed at the outer region of the system at which there 


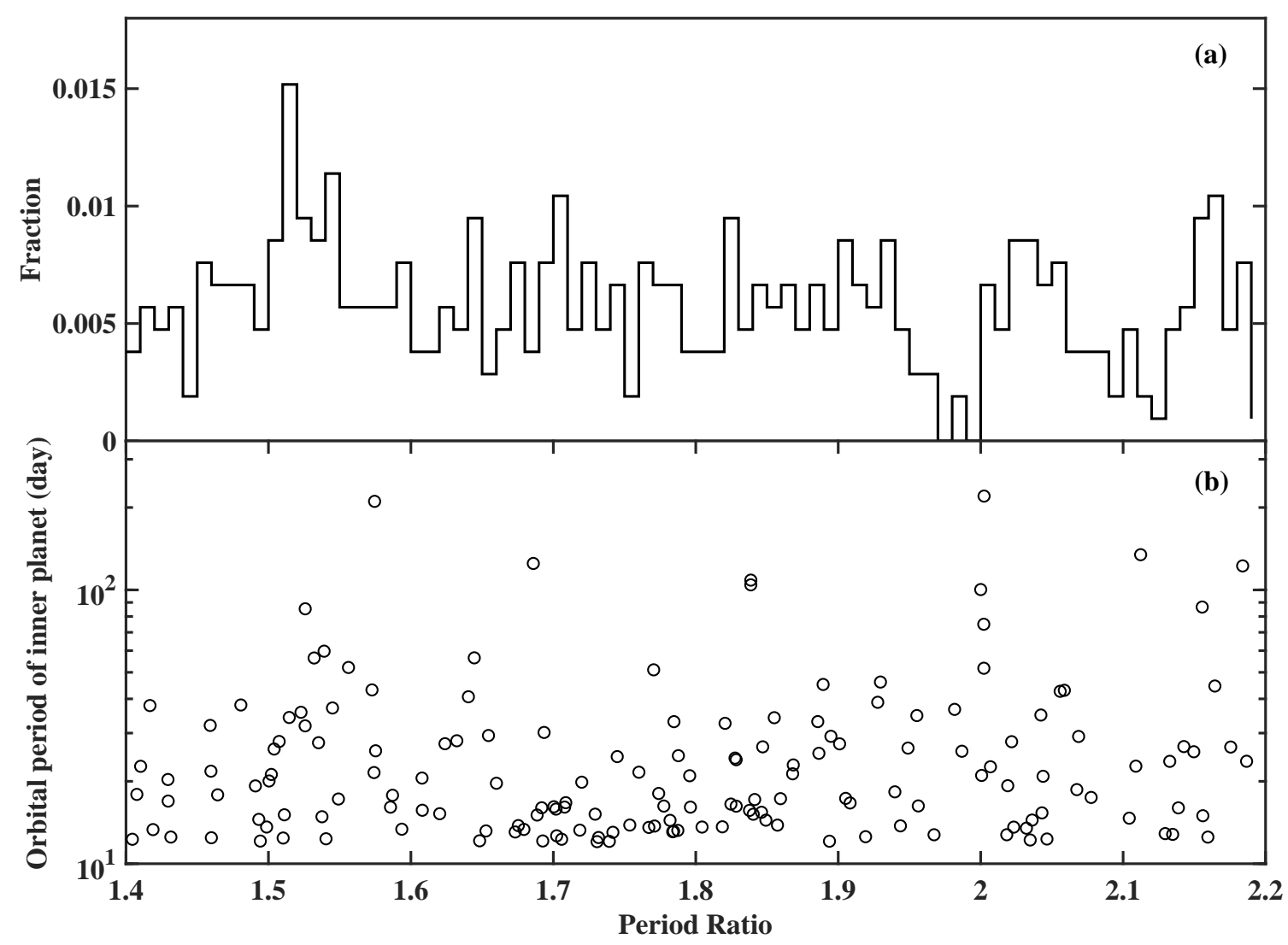

Figure 1. The distribution of period ratio in the system with the innermost planets whose orbital periods are larger than 10 days. Panel (a) shows the fraction of different period ratio in the range of [1.4, 2.2]. Panel (b) displays the distribution of the innermost planets versus the period ratio. The orbital periods of the innermost planets are in a range of 10 - 200 days.

are enough material to form terrestrial planets with couple of Earth-mass. Due to the effect between planets and gas disk surrounded, terrestrial planets will undergo orbital migration (Lin \& Papaloizou 1979; Kley \& Nelson 2012). During the convergent migration, planet pairs can be captured into exact MMRs (Lee \& Peale 2002; Papaloizou \& Szuszkiewicz 2005; Pierens \& Nelson 2008). If planets migrate to the inner region of the system, the effect of the tidal raised by the star can destroy the configuration of MMRs and become near MMRs configuration. Based on this formation scenario, we investigated the configuration formation of KOI-152 system with planets in near 4:2:1 MMRs (Wang et al. 2012), the formation of near 2:1 and 3:2 MMRs effected by the properties of star, the speed of type I migration
(Wang \& Ji 2014), the effect caused by the mass accretion process and the possible outward migration (Wang \& Ji 2017), and the existence of giant planets in planetary systems (Sun et al. 2017; Pan et al. 2020).

We analyse the data with the innermost planets in a system farther than $0.1 \mathrm{AU}$. The results are shown in Figure 1 with 1054 planet pairs. We mainly focus on the region near 2:1 and 3:2 MMRs in the range of [ 1.42 .2$]$ in the distribution of period ratio between planet and its adjacent inner planet. Panel (a) shows the fraction distribution. We find that near the exact location of 2:1 MMRs, where a gap is shown between the period ratio of 1.97 and 2.0. Few cases are found in this region. A peak appears at the period ratio larger than 2.0 especially the region between 2.0 and 2.06. The fraction de- 
creases from 0.01 to 0.001 in the region of [2.06 2.13]. Similar tendency is given near the exact location of 3:2 MMRs. Few planet pairs are found between the period ratio of [1.4 1.5]. A peak exists at the period ratio larger than 1.5 and smaller than 1.55. The fraction decreases from 0.015 to 0.004 in the region of [1.55 1.62]. Another peak appears at about 1.69 which is near 5:3 MMR. The fraction between 1.63 and 1.68 remain stable, is consistently lower than 0.09. Panel (b) of Figure 1 shows the distribution of the orbital period of innermost planets versus the period ratio between two adjacent planets. Most of the innermost planets are in the range of [10 100] days, where seven planet pairs with the period of inner planet larger than 100 days. In this work, our primary goal is to investigate the formation scenario of systems with semi-major axis of the inner planet larger than $0.1 \mathrm{AU}$, along with planet pairs in near exact MMRs configuration.

We suppose that planet pairs can be captured into exact MMRs first during the orbital migration process which induced by the gas disk. Then planet pair will migrate in a same pace and eccentricities of planets can be excited after they are in MMRs. The depletion timescale of gas disk is estimated to be million years (Haisch et al 2001). Here, we assume the gas disk surrounding with the star can survive from $10^{5}$ to $10^{7}$ yr (Williams \& Cieza 2017). If the eccentricity damping effect is still strong enough, eccentricities which have been excited in MMR will be damped. Due to the eccentricity damping, the semi-major axis will change a little. Thus, planet pairs will depart from the exact location of MMRs. In this formation scenario, the depletion and structures of gas disks are possible essential factors that influence the final configurations.

The work is structured as follows, in Section 2 , we describe the models including disk models and numerical methods used in our simulations.
The main results of simulations containing some typical cases and the statistical results are summarized in Section 3. Section 4 shows the conclusions and discussions.

\section{MODELS}

\subsection{Disk Models}

The surface density profile of gas disk $\Sigma_{g}$ based on the empirical minimum-mass solar nebular model (MMSN; Hayashi 1981) at a stellar distance $r$ is described as

$$
\begin{aligned}
\Sigma_{\mathrm{g}} & =f_{\mathrm{g}} \Sigma_{0}\left(\frac{r}{1 A U}\right)^{-k} \exp \left(\frac{-t}{\tau_{\text {dep }}}\right) \mathrm{g} \mathrm{cm}^{-2} \\
& =1700 f_{\mathrm{g}}\left(\frac{r}{1 A U}\right)^{-1} \exp \left(\frac{-t}{\tau_{\text {dep }}}\right) \mathrm{g} \mathrm{cm}^{-2}
\end{aligned}
$$

where $\tau_{\text {dep }}$ is the gas depletion timescale and $f_{\mathrm{g}}$ is the enhancement factor of the MMSN, $k=1$ is the power-law index of the gas density we adopted in this work, and $t$ means the time.

\subsection{Type I migration and gas damping}

We adopt the prescription for type I migration as described in Paardekooper et al. (2010); Paardekooper, Baruteau, \& Kley (2011) where the total torque can be expressed in terms of

$$
\begin{gathered}
\Gamma_{t o t}=f_{t o t} \Gamma_{0} / \gamma \\
f_{t o t}=f_{L B}+f_{C R}
\end{gathered}
$$

where $f_{\text {tot }}, f_{L B}$ and $f_{C R}$ are the coefficients for the total, Linblad, and corotation torques, respectively, $\gamma=1.4$ is the adiabatic index, and

$$
\Gamma_{0}=(q / h)^{2} \Sigma_{p} r_{p}^{4} \Omega_{p}^{2}
$$

where $\Sigma_{p}$ and $\Omega_{p}$ are the disk surface density and angular frequency at the location of the planet $r_{p}, q=M_{p} / M_{*}$, and $h=H / r$ is the disk's aspect ratio at $r_{p}$. The magnitude of $f_{\text {tot }}$ is a function of $s \equiv \partial \ln \Sigma / \partial r, \beta \equiv \partial \ln T / \partial r, q, \nu$ (viscosity), and $\xi$ (thermal diffusivity). Herein, we assume an expression to approximate the $f_{\text {tot }}$ as 


$$
\begin{aligned}
f_{t o t} & =f_{n s c} Q(q)+f_{L B} \\
f_{n s c} & =\operatorname{coe} f \times\left(1-\frac{2\left(\frac{r}{r_{t}}\right)^{2}}{1+\left(\frac{r}{r_{t}}\right)^{2}}\right) \\
Q(q) & =\frac{\left(\frac{q}{q_{1}}\right)^{2}\left(\frac{q}{q_{2}}\right)^{-2}}{2\left(\frac{q}{q_{1}}\right)^{2}\left(\frac{q}{q_{2}}\right)^{-2}+\left(\frac{q}{q_{1}}\right)^{2}+\left(\frac{q}{q_{2}}\right)^{-2}} \\
f_{L B} & =1-\frac{2\left(\frac{r}{r_{m}}\right)^{4}}{1+\left(\frac{r}{r_{m}}\right)^{4}}
\end{aligned}
$$

where $r_{t}$ is transition radius between the outer optically thick region and the inner optically thin disk, it decreases with the mass accretion rate, gas density and time. $r_{m}$ is magnetosphere radius. $f_{n s c}$ is the coefficient of the fully non saturated component of the corotation torque, the saturation parameter $Q(q)$ represents a range of $q_{1}<q<q_{2}$ over which the corotation torque is not saturated. coef is coefficient of $f_{n s c}$. With different coef, the strength of the corotation torque will change.

If $f_{\text {tot }}$ is negative, planet will undergo inward migration with the negative torque. On the contrary, if $f_{\text {tot }}$ is positive, the planet will suffer from outward migration with the positive torque. Figure 2 shows the value of $f_{\text {tot }}$ changing with the mass of planets and the distance away from the central star. If the planet is massive enough $\left(m>M_{\text {crit }}\right)$ (Ida \& Lin 2008),

$$
M_{\text {crit }} \simeq 30\left(\frac{\alpha}{10^{-3}}\right)\left(\frac{a}{1 A U}\right)^{1 / 2}\left(\frac{M_{*}}{M_{\odot}}\right) M_{\oplus},
$$

a gap will form around it. The torque on planet is non-linear due to gap opening and planet will undergo type II migration other than type I migration. $M_{\text {crit }}$ is about $30 M_{\oplus}$ for the system with solar-like star. In Figure 2, the grey dash line shows the location of $30 M_{\oplus}$. The torque we estimated in this work is suitable for the region below the grey line. In Figure 2, red color means that $f_{\text {tot }}$ is positive, while blue color indicates that $f_{\text {tot }}$ is negative. As observed from Figure 2 , we can conclude that planets will experience outward migration within $0.25 \mathrm{AU}$ when the planet mass is about $10 M_{\oplus}$. Here we define this boundary between inward and outward migration as $r_{\text {boundary }}$, which $r_{\text {boundary }}$ changes with the transition radius $r_{t}$. Meanwhile, $r_{\text {boundary }}$ is related to the planet mass. In Figure 2, we assume $r_{t}=0.5 \mathrm{AU}$, coef $=5, q_{1}=3 \times 10^{-5}$ $M_{\odot}$ and $q_{2}=10^{-3} M_{\odot} . r_{\text {boundary }}$ appears to remain between 0.2 to $0.32 \mathrm{AU}$ when the planetary mass ranges from 10 to $300 M_{\oplus}$ as shown in the blue line. The timescale of type I migration is

$$
\tau_{a}=\frac{m_{p} \sqrt{G M_{*} r}}{2 \Gamma_{\text {total }}}=\frac{m_{p} \gamma \sqrt{G M_{*} r}}{2 f_{a}\left(\frac{q}{h}\right)^{2} \Sigma_{g} r^{4} \Omega^{2}}=\frac{\tau_{0}}{f_{a} h^{2}} .
$$

Herein, $f_{a}=f_{\text {tot }}$. The timescale of gas damping is

$$
\tau_{e}=h^{2} f_{a} \tau_{a}=\frac{m_{p} h^{2} \gamma \sqrt{G M_{*} r}}{2\left(\frac{q}{h}\right)^{2} \Sigma_{g} r^{4} \Omega^{2}}=\tau_{0} .
$$

where $M_{*}$ represents the mass of central star and we choose $M_{*}=1 M_{\odot}$ in this work. $\Omega$ is the Kepler angular velocity, and $G$ is the gravitational constant.

The force of eccentricity damping is expressed as

$$
\mathbf{F}_{d a m p}=-\frac{(\mathbf{v} \cdot \mathbf{r}) \mathbf{r}}{r^{2} \tau_{e}}
$$

The force of type I migration is

$$
\mathbf{F}_{\text {migI }}=\frac{\Gamma_{t o t}}{m_{p} r}
$$

The acceleration of the planetary embryos with a mass $m_{i}$ is described as

$$
\begin{aligned}
& \frac{d}{d t} \mathbf{V}_{i}=-\frac{G\left(M_{*}+m_{i}\right)}{r_{i}{ }^{2}}\left(\frac{\mathbf{r}_{i}}{r_{i}}\right) \\
& +\sum_{j \neq i}^{N} G m_{j}\left[\frac{\left(\mathbf{r}_{j}-\mathbf{r}_{i}\right)}{\left|\mathbf{r}_{j}-\mathbf{r}_{i}\right|^{3}}-\frac{\mathbf{r}_{j}}{r_{j}^{3}}\right] \\
& +\mathbf{F}_{\text {damp }}+\mathbf{F}_{\text {migI }} \text {, }
\end{aligned}
$$




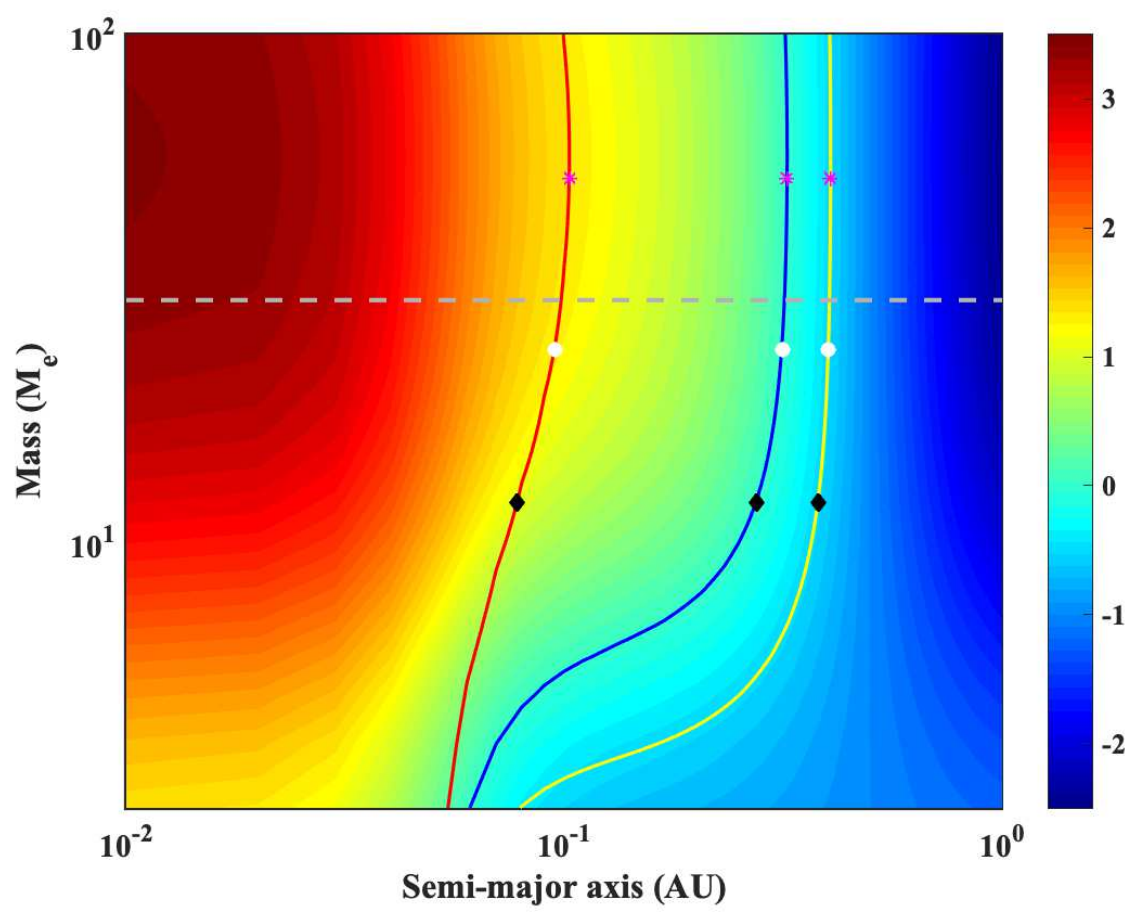

Figure 2. The contour of total torque $f_{\text {tot }}$ varying with the planetary mass and semi-major axis. The colorbar index denotes the values of the total torque, where red color represents the positive torque while the blue stands for the negative torque. If the semi-major axis is less than $r_{\text {boundary }}$, planets will undergo outward migration. The red, blue and yellow lines represent the location of $r_{\text {boundary }}$ with respect to coe $f=2$, coef $=5$ and coef $=10$, respectively. The black diamonds show the exact locations of $r_{\text {boundary }}$ for the planets with $12 M_{\oplus}$ which is the mass of the inner planet in our simulations, while the white dots display the exact locations of $r_{\text {boundary }}$ for the planets with $24 M_{\oplus}$ which is the mass of the outer planet in our simulations. The magenta stars represent the furthest location on each boundary line, it locates at $0.1 \mathrm{AU}$ for $\operatorname{coe} f=2,0.32 \mathrm{AU}$ for coef $=5$, and $0.41 \mathrm{AU}$ for coe $f=10$, respectively.

In this work, we integrate equation (13) to explore the dynamical evolution of planets in the system using the time symmetric integrator Hermit scheme (Aarseth 2003). In our numerical simulations, all planets are initially assumed to occupy coplanar and circular orbits. The mean anomaly and the argument of pericenter are generated between $0^{0}$ to $360^{\circ}$ randomly. Each case is integrated to $10 \mathrm{Myr}$.

\section{NUMERICAL SIMULATION RESULTS}

In our model, we consider the planetary systems are composed of two planets with a couple of Earth-mass and a solar-mass central star. Herein we perform extensive numerical simulations to investigate the dynamical evolution of planet pairs in the system, where we take into account the combined parameters of a wide variety of density of gas disk $\Sigma_{g}$ which reflects the change of $f_{g}$, the depletion timescale of gas disk $\tau_{\text {dep }}$, the coefficient of the torque coe $f$, the transition radius $r_{t}$, the disk aspect ratio $h . p_{10}$ and $p_{20}$ are the initial orbital periods of two planets, the subscripts 1 and 2 represent the values of inner planet $P_{1}$ and outer planet $P_{2}, m_{1}$ and $m_{2}$ respectively denote the planetary masses. This allows us to extensively explore the dynamical nature of planet pairs at specific MMRs using the above-mentioned parameters.

We entirely carry out 288 runs in two groups with the combination parameters as abovementioned. The details of parameters we 

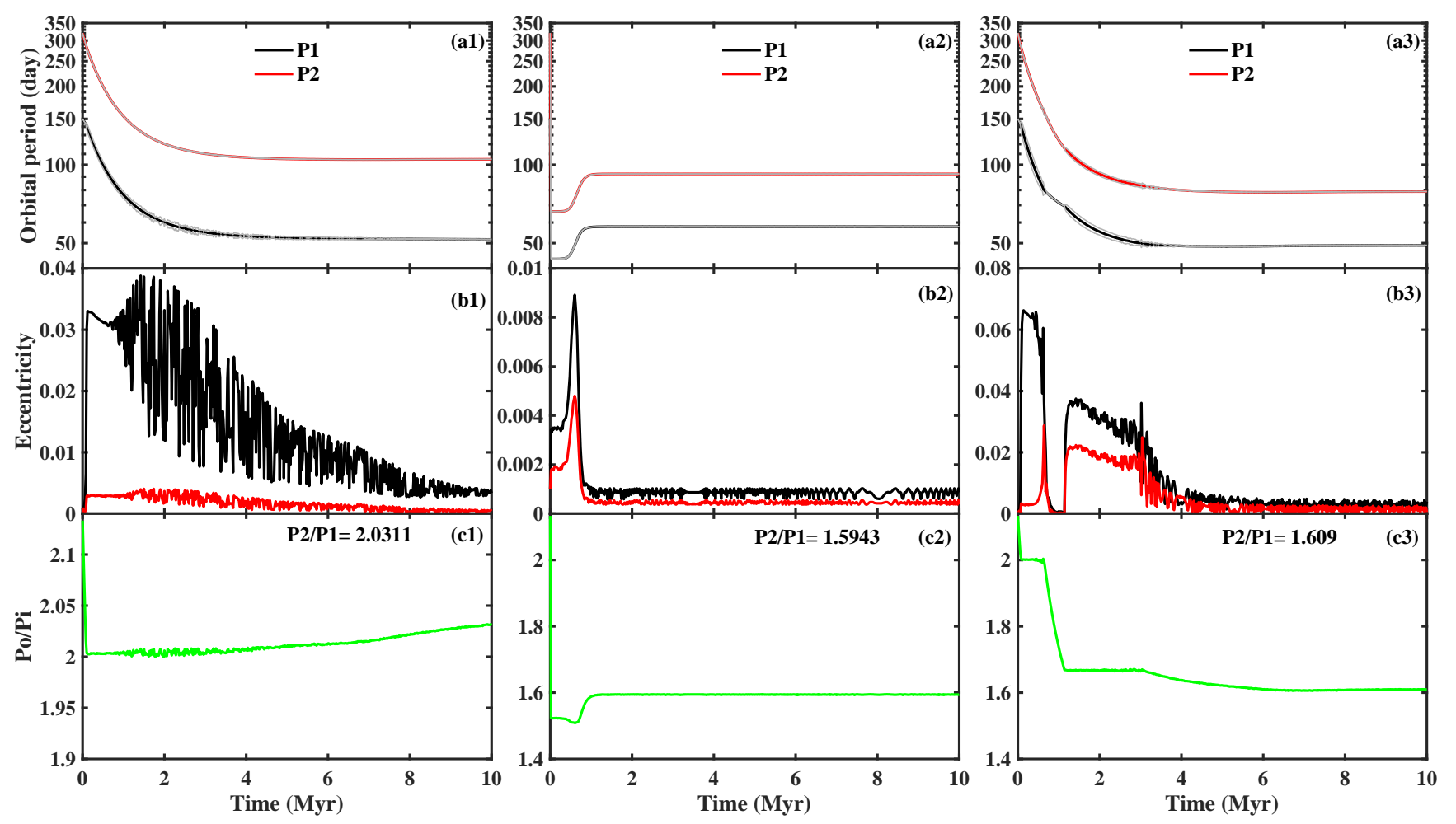

Figure 3. The evolution of Case 1-3. Three typical runs show that two planets finally deviates from exact MMR. Panel (a1-3), (b1-3), and (c1-3) display the evolution of the orbital periods, semi-major axes, eccentricities, and the period ratio, respectively. The black line and red lines, respectively represent the evolutions of orbital periods for the inner planet $\left(P_{1}\right)$ and the outer planet $\left(P_{2}\right)$. In Panel (a), the grey lines are associated with the evolution of the pericenter and apocenter, respectively.

adopted in each group are shown in Table 1 . In the following, we show five typical runs (Cases 1-5) revealing the evolution scenario that two planets deviate from exact MMRs. Table 2 summarizes the adopted initials for three runs, where $p_{1 e}$ and $p_{2 e}$ represent the resultant orbital periods of the pairs, respectively.

\subsection{Escape process from the exact location of $M M R$}

After two planets are trapped in exact location of MMR during orbital migration, they will keep migrating in a same pace. The eccentricities of them can be excited in this process. Due to the existence of gas disk, eccentricities can be damped leading to the change of orbital period at the same time. The change of angular momentum caused by the eccentricity damping force is zero, therefore, we can get that

$$
\Delta a \propto a e \Delta e /\left(1-e^{2}\right) \simeq a e^{2},
$$

where $\Delta a$ is the change of semi-major axis caused by the eccentricity damping force. If eccentricities of two planets are excited to comparable values, the change of semi-major axes $\Delta a$ of them are mainly proportional to $a$. The outer planet will change larger distance than the inner one due to the eccentricity damping process. If the eccentricity of inner planet is excited to value which is much larger than the outer one, the change of period ratio is related to $a e^{2}$ which depends on the relative space between two planets and the amplitude of eccentricity after they are excited. 
Table 1. The parameters used in two groups. $p_{10}$ and $p_{20}$ are the initial orbital period of two planets in the systems. $m_{1}$ and $m_{2}$ are the masses of planets. The main difference between group 1 and 2 is the transition radius $r_{t}$.

\begin{tabular}{|c|c|c|c|c|c|c|c|c|c|}
\hline Group & $\begin{array}{l}\tau_{d e p} \\
\mathrm{Myr}\end{array}$ & $\begin{array}{c}r_{t} \\
(\mathrm{AU})\end{array}$ & coef & $h$ & $f_{g}$ & $\begin{array}{c}p_{10} \\
\text { (day) }\end{array}$ & $\begin{array}{c}p_{20} \\
\text { (day) }\end{array}$ & $\begin{array}{c}m_{1} \\
\left(M_{\oplus}\right) \\
\end{array}$ & $\begin{array}{c}m_{2} \\
\left(M_{\oplus}\right)\end{array}$ \\
\hline 1 & $0.1,0.5,1,3$ & 0.5 & $2,5,10$ & $0.02,0.05,0.1,0.05 r^{1 / 4}$ & $1 / 50,1 / 10,1$ & 150 & 320 & 12 & 24 \\
\hline 2 & $0.1,0.5,1,3$ & 0.2 & $2,5,10$ & $0.02,0.05,0.1,0.05 r^{1 / 4}$ & $1 / 50,1 / 10,1$ & 150 & 320 & 12 & 24 \\
\hline
\end{tabular}

Table 2. The parameters used in four cases. $p_{10}$ and $p_{20}$ are the initial orbital period of two planets in the systems. $m_{1}$ and $m_{2}$ are the masses of planets. $p_{1 e}$ and $p_{2 e}$ represent each of the final orbital periods, respectively. $f_{g}$ is enhance factor of the standard gas disk.

\begin{tabular}{cccccccccccc}
\hline Case & $\begin{array}{c}\tau_{\text {dep }} \\
\mathrm{Myr}\end{array}$ & $\begin{array}{c}r_{t} \\
(\mathrm{AU})\end{array}$ & coef & $h$ & $f_{g}$ & $\begin{array}{c}P_{10} \\
(\text { day })\end{array}$ & $\begin{array}{c}P_{20} \\
(\text { day })\end{array}$ & $\begin{array}{c}m_{1} \\
\left(M_{\oplus}\right)\end{array}$ & $\begin{array}{c}m_{2} \\
\left(M_{\oplus}\right)\end{array}$ & $\begin{array}{c}P_{1 e} \\
(\text { day })\end{array}$ & $\begin{array}{c}P_{2 e} \\
(\text { day })\end{array}$ \\
\hline 1 & 1 & 0.5 & 2 & 0.05 & $1 / 50$ & 150 & 320 & 12 & 24 & 51.69 & 104.99 \\
2 & 0.1 & 0.5 & 5 & 0.02 & $1 / 1$ & 150 & 320 & 12 & 24 & 57.85 & 92.23 \\
3 & 1 & 0.5 & 2 & 0.1 & $1 / 10$ & 150 & 320 & 12 & 24 & 49.03 & 78.89 \\
4 & 1 & 0.5 & 5 & 0.05 & $1 / 500$ & 300 & 540 & 24 & 12 & 245.70 & 495.58 \\
5 & 1 & 0.5 & 5 & 0.05 & $1 / 500$ & 300 & 640 & 12 & 24 & 264.57 & 529.61 \\
\hline
\end{tabular}

\subsection{Case 1: Departure from the Exact Location of 2:1 MMR}

Panel (a1), (b1) and (c1) of Figure 3 show a typical run for two planets departing from 2:1 MMR. Here the depletion timescale of gas disk is assumed to be $1 \mathrm{Myr}$ and the gas density is fiftieth of the standard MMSN model. According to description of surface density of gas disk in equation (1), $\Sigma_{g}$ will decrease to $\Sigma_{0} / e$ at 1 Myr at the same location, but the gas is still exist and eccentricity damping effect is working in the next few million years. As noted from Figure 3, two planets undergo an inward type I migration before the inner planet reaches $\sim 50$ days at about $2 \mathrm{Myr}$. The planet pair is captured into exact 2:1 MMR quickly. Planets will remain at the exact location of 2:1 MMR if there is no eccentricity damping exist. According to the estimation of equilibrium eccentricity in MMR (equation (A27) of Papaloizou \& Szuszkiewicz (2005)), the amplitude of eccentricity is affected by the mass ratio, period ratio and the ratio between timescale of eccentricity damping and orbital migration (Murray \& Dermott 1999; Nelson \& Papaloizou 2002; Papaloizou \& Szuszkiewicz 2005; Liu et al. 2015). Eccentricity tends to be excited to higher value for less massive inner planet. In case 1 , in the first two million years, the eccentricity of the inner planet can be excited to be approximately 0.04 , whereas that of the outer planet is not radically stirred up but performs a slight fluctuation about 0.005. In Panel (b1) of Figure 3, we observe that the eccentricity of $P_{1}$ falls down to 0.01 gradually due to the eccentricity damping from 2 to $4 \mathrm{Myr}$. In this case, the excited eccentricity of $P_{1}$ when two planets are trapped into exact 2:1 MMR is much higher than that of $P_{2}, e_{1} / e_{2} \sim 10$, while $a_{1} / a_{2}$ almost keeps in 0.63 for 2:1 MMR. Thus, according to equation (14), $\Delta a_{1}$ is larger than $\Delta a_{2}$ with the damping of eccentricities, the space between two planets become larger 
and larger. Panel (c1) of Figure 3 displays the evolution of the ratio of orbital period between two planets $P_{2} / P_{1}$, which was started at about 2.13 (not in 2:1 MMR), then temporarily to be 2.0 (captured into exact 2:1 MMR with resonant angles librating in very small amplitude), and a final value of 2.0317 (leaving out of exact location of 2:1 MMR with resonant angles librating in large amplitude). Clearly, this gives a dynamical portrait for elucidating the resonant evolution history for two planets in the system. As given in Table 2, we have their resultant orbital periods of 51.69 and 104.99 days, respectively, being suggestive of that two planets do slightly deviate from exact 2:1 MMR.

From the Kepler data, we can present several systems which hold planets near the exact location of 2:1 MMRs. Such as the systems Kepler328 and Keper-56. Kepler-328 (Xie 2014) horbors two planets confirmed in 34.92 and 71.31 days, the period ratio is 2.0421. Kepler-56 (Steffen \& Hwang 2015) owns two planets in 10.50 and 21.41 days, with an orbital period ratio of 2.0390. These two systems may be formed through the similar scenario as Case 1 describes.

\subsection{Case 2: Departure from the Exact Location of 3:2 MMR}

The parameters used in this case are shown in the second line of Table 2. Comparing with Case 1, the density of the gas disk is larger in this case, using the standard MMSN model, and the coefficient of $f_{n s c}$ is 5 which is larger than that of Case 1. Therefore, the boundary $r_{\text {boundary }}$ is much larger in Case 2 than in Case 1. The red, blue, and yellow lines in Figure 2 show the distribution of $r_{\text {boundary }}$ with coe $f=2$, coef $=5$ and coef $=10$, respectively. At the boundary location, the coefficient of total torque is 0 , and when planet approaches the boundary, the speed of orbital migration will be reduced. We note that the boundary line declines as coef decreases. For the inner planet which holds $12 M_{\oplus}, r_{\text {boundary }}$ moves from $\sim 0.08$
AU (corresponding to the orbital period of 8.5 days) to $0.27 \mathrm{AU}$ (corresponding to the orbital period of 51 days) when we change coef from 2 to 5 as shown in the black diamonds in Figure 2. $r_{\text {boundary }}$ is the largest when coef $=10$, the boundary locates at about 0.38 AU (corresponding to the orbital period of 85 days). For the outer planet with mass in $24 M_{\oplus}, r_{\text {boundary }}$ moves from $\sim 0.1 \mathrm{AU}$ ( 10 days) with coef $=2$ to $0.32 \mathrm{AU}$ (66 days) with coe $f=5$ as shown in white dots in Figure 2. Therefore, it seems to be possible for inner planet whose semi-major axis is smaller than $0.27 \mathrm{AU}$ to migrate outward in Case 2.

The evolution process of Case 2 is shown in panel (a2), (b2), and (c2) of Figure 3. In such a case, $f_{g}=1$, the gas density is fifty times of the value given in Case 1, suggesting a much faster inward orbital migration. As a result, this contributes to the major reason inducing two planet passed through 2:1 MMR and trapped into 3:2 MMR. When two planets are in the location near 2:1 MMR, the torque on the planet is still strong enough, thus they will continue migrating inward until they stop at the region near 43.8 and 66.6 days, respectively. Different with Case 1, the inner planet run through the location of $r_{\text {boundary }}$ to the inner region which may cause outward orbital migration. Within $0.9 \mathrm{Myr}$, they are kept in the exact location of 3:2 MMR with small amplitude of resonant angles as shown in panel (c2) of Figure 3. The eccentricities of inner planet can be excited to be about 0.01. Subsequently, decrease of semimajor axis is caused due to eccentricity damping. According to equation (14), the variation of semi-major axis of $P_{1}$ caused by eccentricity damping is larger than that of $P_{2}$. Considering outward orbital migration and the effect of eccentricity damping simultaneously, two planets migrate outward in $0.1 \mathrm{Myr}$, and the outer planet migrate outward for a longer distance than the inner planet. Finally, two planets even- 
tually arrive at 57.85 and 92.23 days respectively with a ratio of orbital period 1.5943 which deviates from the exact location of 3:2 MMR with large amplitude resonant angles. This indicates a scenario of planet pair departs from the exact location of 3:2 MMR owing to eccentricity damping and outward orbital migration.

Kepler-276 and Kepler-279 (Rowe et al. 2014) are the systems with planets near the exact location of 3:2 MMRs. There are three planets confirmed in both systems. Planets locate at 14.13, 31.88 , and 48.65 days in Kepler-276. The outer two planets are in near 3:2 MMRs. The period ratio of them is about 1.5260 . The configuration of Kepler-279 is similar to Kepler-276. Three planets are in 12.31, 35.74, and 54.42 days, respectively. The period ratio of the outer two planets is about 1.5227 . The formation process showed in case 2 may be a possible explanation about the formation of these two systems.

\subsection{Case 3: Departure from the Exact Location of 5:3 MMR}

Compared with Case 1, we adopt a disk aspect ratio $h=0.1$ and the gas density herein $f_{g}=$ 0.1 , being tenth of the standard MMSN model in Case 3. According to Equation (9) and (10), the migration timescale $\tau_{a}$ in Case 3 is 0.8 times of that in Case 1 and the eccentricity damping timescale $\tau_{e}$ in Case 3 is 3.2 times of that in Case 1. Thus, the eccentricity damping process can exist for longer time in Case 3.

Similarly with Case 1 , we remain coef $=2$ in the calculation. $r_{\text {boundary }}$ locates at about $0.08 \mathrm{AU}$ for inner planet and $0.095 \mathrm{AU}$ for outer planet. Panel (a3), (b3), and (c3) of Figure 3 show the evolution of two planets. From the evolution of period ratio in Panel (c3), we note that two planets are trapped into 2:1 MMR at the very beginning. With faster speed of orbital migration, two planets break through 2:1 MMR and perform continuous migrating into the inner region, and they will be further captured into 5:3 MMR with resonant angles librating at very small amplitude at $\sim 1$ Myr. Since the inner planet is less massive, the eccentricity of it should be excited to larger value than the outer one . However, the ratio of eccentricity between the outer and inner planet $e_{2} / e_{1}$ increases with the decrease of $a_{2} / a_{1}$ (Nelson \& Papaloizou 2002; Papaloizou \& Szuszkiewicz 2005). Therefore, in this case, eccentricities of both planets can be stirred up to comparable values, about 0.03 . Under such circumstance, both planets smoothly migrate when the eccentricities damp down. And at this time, two planets locate at the outer region of $r_{\text {boundary }}$. Therefore, they will migrate inward. Due to the similar $e$ and $\Delta e, \Delta a$ is proportion to $a$ according to equation (14). Thus, the outer planet will move inward with longer distance than the inner planet. The simulation results are consistent with the theoretical estimation. Consequently, the final ratio of their orbital periods is 1.6099 as shown in panel (c3) of Figure 3, implying that two planets move a little out of 5:3 MMR. In the end, two planets halt migration and remain at the orbits of 49.03, and 78.89 days, respectively. Planet pair is out of MMR with cicular resonant angles.

The system Kepler-197 (Rowe et al. 2014), which holds four planets, has planet pair in the configuration similar to that shown in Case 3. Planet $\mathrm{d}$ and e locate at about 15.68 and 25.21 day which make the period ratio of them equal to 1.6078. The system Kepler-154 has planet pair in near 5:3 MMRs. There are five planets in the system (Rowe et al. 2014; Morton et al. 2016). The third planet, Kepler-154 d, and the fourth planet, Kepler-154 b, are in the period of 20.55 and 33.04 day, respectively. Their period ratio is about 1.6078, which is the same as the planet pair in Kepler-276. These systems may share the similar formation scenario with Case 3 . 


\subsection{Case 4: Departure from the Exact Location of 2:1 MMR with Divergent Migration}

The systems in Group 1 and 2 are initially set to have two planets and the mass of outer planet is larger than the inner one. Base on the estimation of isolation mass of planet embryos (Ida \& Lin 2004), $m$ is proportional to $a^{3 / 4}$. The outer planet tends to contain more material than the inner one. According to the statistics on the Kepler candidates (Wang \& Ji 2017), there is opportunity for stars to bear a larger innermost planet in a system. Herein, we run a case on the system with higher mass inner planet to test the effect of the eccentricity damping.

In this case, the mass of the inner planet is $24 M_{\oplus}$, and the mass of the outer one is 12 $M_{\oplus}$. The results are shown in Figure 4. The transition radius is $0.5 \mathrm{AU}$, the scale heigh of disk is 0.05 , coef $=5, f_{g}=0.002$ and the depletion timescale of the gas disk is $1 \mathrm{Myr}$. Details of the initial settings in this case are shown in Table 2 as case 4 . Due to the low gas density of the disk, the migration process is very gentle. The initial periods of two planets are 300 and 540 day, respectively. The period ratio of them is smaller than 2.0 initially. After about 3 Myr divergent migration, the period ratio of planet pair will increase from 1.8 to 2.0, two planets are trapped into 2:1 MMRs, the resonant angle librate with small amplitude as shown in panel (d1) of figure 4. Since the inner planet is more massive, the eccentricity of it should be excited to smaller value than the outer one. However, $e_{2} / e_{1}$ is also related to $g=\left(m_{1} a_{2}\right) /\left(m_{2} a_{1}\right)$ (Nelson \& Papaloizou 2002; Papaloizou \& Szuszkiewicz 2005). With increase of $g, e_{2} / e_{1}$ decreases. $g$ in case 4 is larger than that in case 1 . Thus, eccentricities of two planets become more comparable in case 4 than in case 1 . The eccentricities of them can be excited to be 0.03 as shown in Panel (c) and the eccentricity of the inner planet is a little higher than that of the outer one. Then the eccentricities can be damped due to the gas disk and finally the period ratio of planet pair can reach 2.0175, which is slightly larger than 2.0. The resonant angles become circular, planet pair is out of MMR as shown in panel (d1) of figure 4. Comparably, we run case 5 with similar initial conditions as in case 4 , but switch the order of the two planets. The initial conditions are shown in Table 2 and the evolution process is shown in figure 4. Two planets are captured into 2:1 MMR at about 1 Myr. Eccentricity of the inner planet can be excited to about 0.05 . The 2:1 MMR is more robust through convergent migration than through divergent migration, thus eccentricity damping effect with lower gas density $\left(f_{g}=1 / 500\right)$ cannot make the planet pair in case 5 leave the exact location of MMR, but can destroy the MMR configuration in case 4 .

According to our formation scenario, systems which hold a larger inner planet than the outer one can form the configuration of near MMRs. Noticeably, two planets locate at about $0.8 \mathrm{AU}$ and $1.2 \mathrm{AU}$ at the end of the simulation. This case can explain the systems near MMRs configuration with the innermost planet quite far away from the central star. Additionally, if planets locate in the inner region of the system initially where planets will undergo outward migration, they can also get out of the exact MMR to be near MMR. The process is similar to that as shown in Case 1-3. The difference is that with outward orbital migration, the innermost planet can reach the location farther away from the central star. Considering all the cases in this work, with the eccentricity damping of the gas disk, planet pairs can get out of the exact MMRs to be the configuration of near MMRs.

\subsection{Statistical Results}

We entirely perform a set of 288 simulations by considering a combination of gas density, coefficient, timescales of gas disk, the disk aspect 

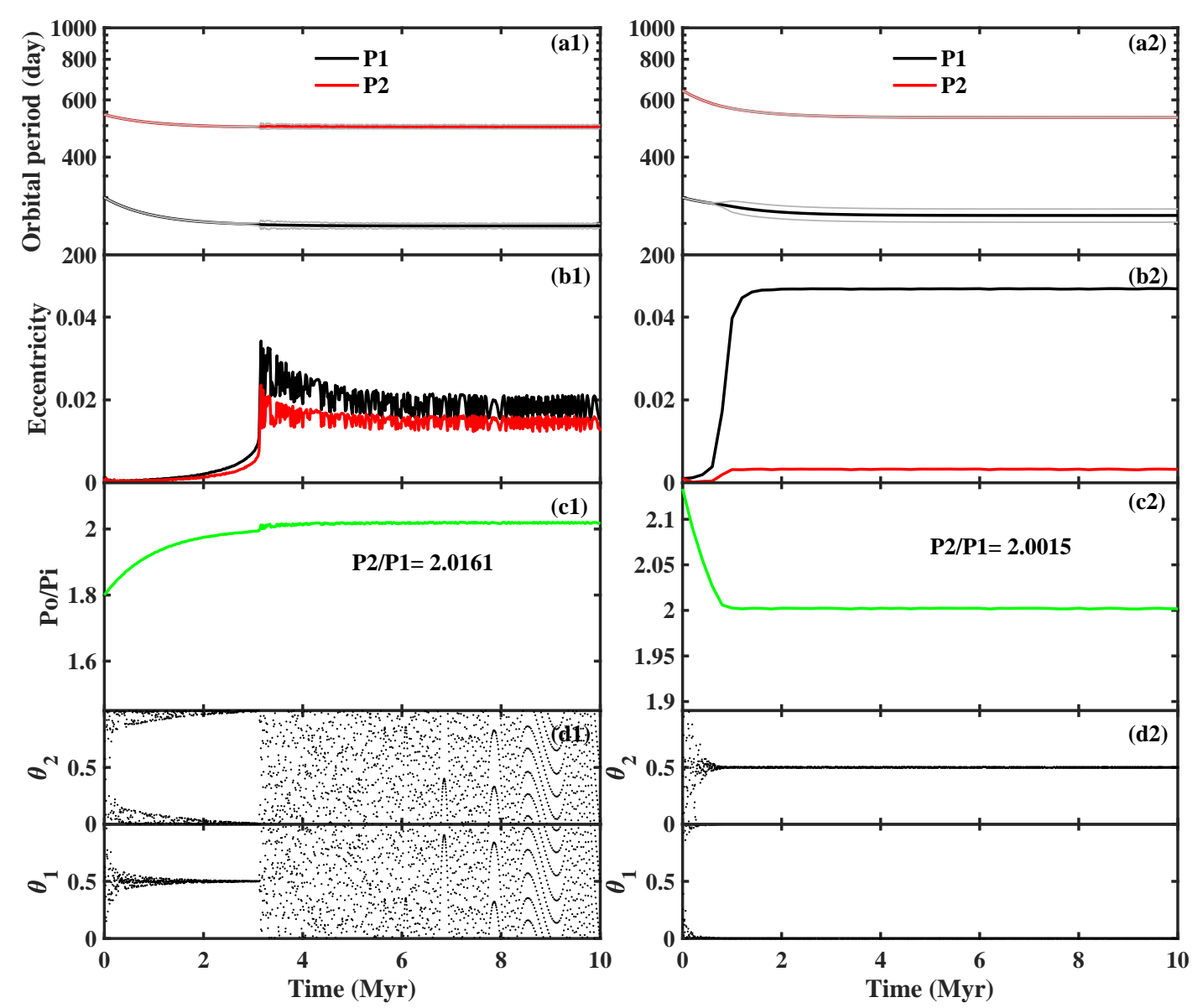

Figure 4. The evolution of case 4 and 5. Panel (a1) and (a2) show the evolution of orbital periods, panel (b1) and (b2) display the evolution of eccentricity, panel (c1) and (c2) represent the evolution of period ratio between two planets of each case, panel (d1) and (d2) show the evolution of resonant angles, where $\theta_{1}=\left(2 \lambda_{2}-\lambda_{1}-\varpi_{1}\right) / 2 \pi, \theta_{2}=\left(2 \lambda_{2}-\lambda_{1}-\varpi_{2}\right) / 2 \pi, \lambda$ and $\varpi$ are the mean longitude and the longitude of pericentre of each planet, respectively.

ratio, and transition radius. The detailed initial settings are given in Table 1. Figure 5 shows the distribution of the period ratio between two planets at the end of the simulations. Panel (a) displays the fraction of period ratio in the range of [1.4 1.8], which means that two planets are in near 3:2 and 5:3 MMRs. Panel (b) exhibits the fraction of planet pairs in near 2:1 MMR, where the period ratio is in the range of [1.95 2.2]. From Figure 5, we find that two peaks appear to be near 3:2 and 2:1 MMRs obviously. Particularly, there is a deficit of planet pairs with period ratio smaller than exact 2:1 and 3:2 MMRs, an excess of pairs with period ratio larger than $2: 1$ and 3:2 MMRs. The results are consistent with the observational distribution shown in Figure 1 (Lissauer et al. 2011). Another small peak appears at the position where the period ratio larger than 1.66 slightly. The fraction of period ratio between 1.5 and 1.66 experiences a decrease from $8 \%$ to $1 \%$, while the fraction of period ratio larger than 2.0 see a decrease from $15 \%$ to $1 \%$. These trends can be seen from the observation data in Figure 1. From table 1, we know that the initial periods of two planets are 150 and 320 day. The period ratio between them is about 2.13, which is larger than 2.0. Therefore, planet pairs are trapped into $2: 1$ MMR first and more easier to be kept in near 2:1 MMR, leading to the absence of data be- 
tween 1.77 and 2.0 which is inconsistent with the observation result. With the same reason, few systems in our simulations hold planets with period ratio lager than 2.1. In summary, the distribution of period ratio which obtained through the formation scenario we proposed is consistent with the observation results.

Figure 6 and 7 show the distribution of period ratio varies with different parameters of the gas disk. Figure 6 shows the distribution of period ratio between 1.4 and 1.8, while Figure 7 displays the period ratio distribution from 1.95 to 2.2. The period ratios of few cases are less than 1.4 , we choose 1.4 as the minimum period ratio as shown in these figures. Panel (a)-(d) of two figures exhibit the results obtained through 144 runs in Group 1 with $r_{t}=0.5$. The overall trend with data from Group 2 with different disk parameters is similar to that in Group 1. There are 288 dots in Panel (e) with all results from Group 1 and 2, which reveal the major difference with different $r_{t}$.

From Figure 6 and 7, we find that a great number of planet pairs are involved in the position very close to the exact locations of $2: 1$ and 3:2 MMRs, which is consistent with the results shown in Figure 5. From Panel (a) of Figure 6 and 7 , we get that planet pairs are more easily to be in near 3:2 MMR rather than 2:1 MMR with longer disk depletion timescale especially for the system with $\tau=5 \mathrm{Myr}$ labeled in green dots, while more planet pairs are in the configuration near 2:1 MMR with $\tau \leq 0.5 \mathrm{Myr}$. The possibilities that planet pairs in near $3: 2$ and 2:1 MMRs are almost even. The results demonstrate that planet pairs have long time to get rid of the 2:1 MMR which is the first low order MMR they meet. With longer disk depletion timescale, $\tau>1 \mathrm{Myr}$ the period ratio of planet pair is wide spreading in the whole region from 1.5 to 2.15 , most of them in the range of $[1.5$ 1.7] and a small part of them distribute at the period ratio of [2.0 2.15]. With $\tau \leq 0.5 \mathrm{Myr}$, most planet pairs contribute to pipe-up of period ratio near 2.0, especially from 2.0 to 2.02 .

Based on the formation scenario, planet pair is trapped into MMR quickly through orbital migration process and eccentricities of them will be excited, the eccentricity damping effect is still strong enough to work on planets making them depart from the exact location of MMR with longer disk depletion timescale. Therefore, longer disk depletion timescale is more helpful in planet pairs departure from the exact location of MMRs.

In all cases, planets locate less than $1 \mathrm{AU}$, thus $h=0.05 r^{1 / 4}$ is the most smallest one among the $h$ value we chosen. Through Panel (b), we show that, with higher $h$ value, longer timescale of eccentricity damping, planet pairs have more opportunities to escape to the configuration that depart farther from the exact location of MMRs.

Moreover, we notice that most of these cases with coef $=10$ which are marked by blue dots in Panel (c) are in the region with period ratio less than 1.55 (or 2.04 for the planet pair in near 2:1 MMR). With the decrease of coef, planet pairs leave far from their exact region of MMRs.

From equation (9), we learn that $f_{g}$ is related to the density of gas disk which determine the speed of orbital migration directly. With higher gas density, larger $f_{g}$, planet pairs are more easily to break through 2:1 to be captured 3:2 MMR (Wang et al. 2014). Panel (d) shows the results consistent with the estimation. Most of the cases with $f_{g}=1 / 50$ labeled in blue dots distribute near the 2:1 MMR.

Based on our analysis of the torque acted on the planets, with the decrease of the transition radius $r_{t}$, the orbital periods of inner planets will decline. Combined with 144 cases with $r_{t}=0.2$, we can conclude that most of the inner planets lying in the range [20,250] days with $r_{t}=0.5$, while the periods of inner planets can be much closer to 10 days with $r_{t}=0.2$ as shown in Panel (e) of Figure 6 and 7. With the 


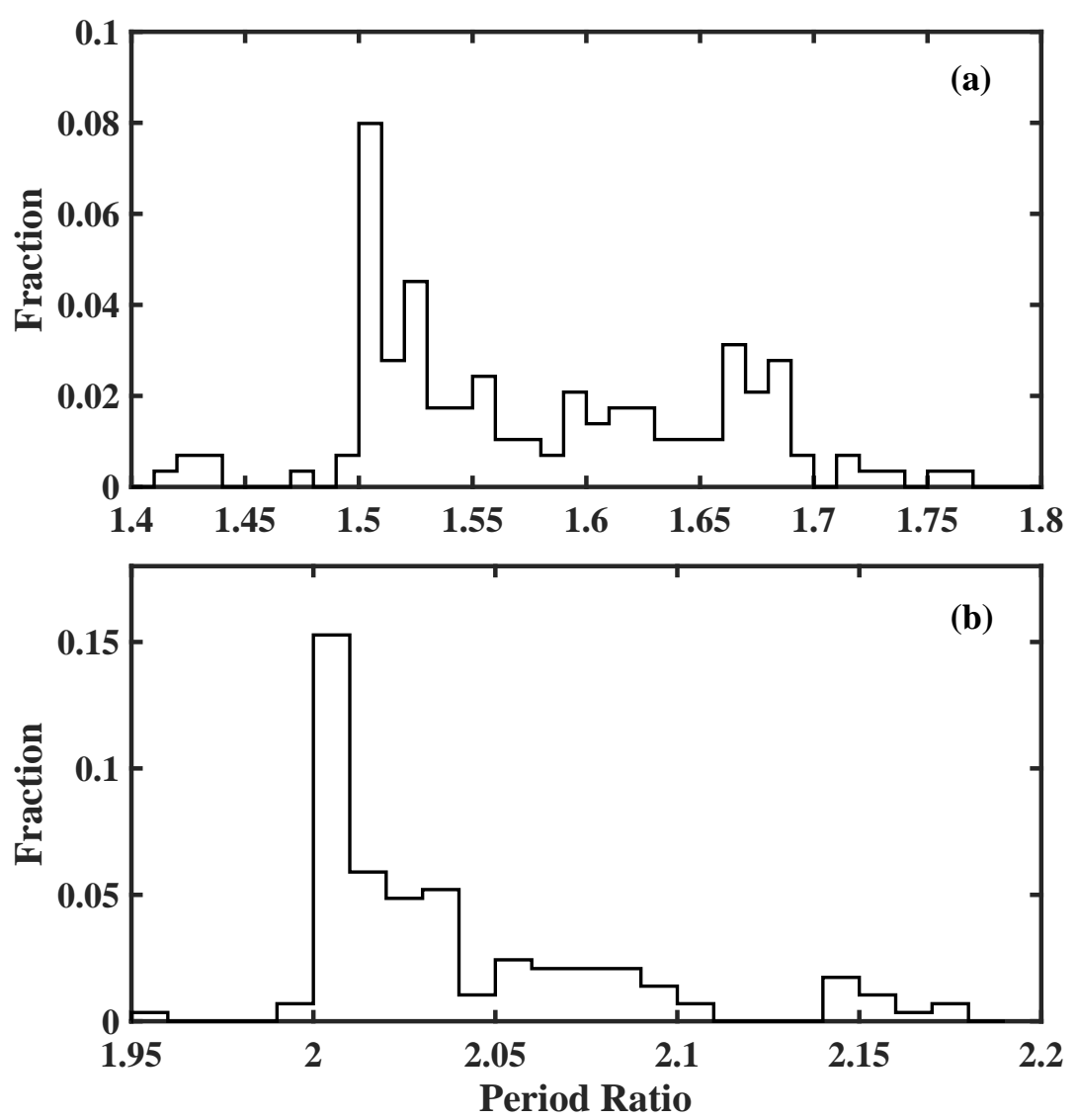

Figure 5. Distribution of period ratio between two planets at the end of the simulations. Panel (a) shows the planet pairs which are in near 3:2 and 5:3 MMRs. Panel (b) displays the distribution of planets locating near 2:1 MMR.

evolution of the star, the transition radius will migrate inward. Therefore, the systems with inner planets lying near 10 days may form at the late stage of the star.

Additionally, from Figure 6 and 7, we can obtain that the resonant offset from 2.0 can extend to almost 2.2 and most planet pairs pipes up in the region $2.0 \leq P_{2} / P_{1} \leq 2.1$. The period ratios of planet pairs which near 1.5 and 1.667 can extend in the range of $[1.3,1.7]$. Only few cases with larger scale height can be maintained in the region between 1.8 and 2.0 .

\section{CONCLUSIONS AND DISCUSSIONS}

In this work, we mainly investigate the formation of planetary systems in the configuration of near MMRs, especially for the systems with the semi-major axis of innermost planets larger than 0.1 AU. Considering the eccentricity damping effect induced by the gas disks, we entirely perform 288 runs of simulations under the condition of a wide variety of depletion timescales of gas disk, the disk aspect ratio, the enhance factor of the gas density, the coefficient of the co-rotation torque, and transition radius of the disk. From the simulations, we conclude that with proper eccentricity damping effect, planet pairs can deviate from perfect MMRs and tune in near MMRs, being indicative of that our proposed scenario is likely to throw light on the distribution nature of the observed systems. Here we summarize the major results as follows:

1. According to our formation scenario, planet pairs can be trapped into $2: 1,3: 2$ 


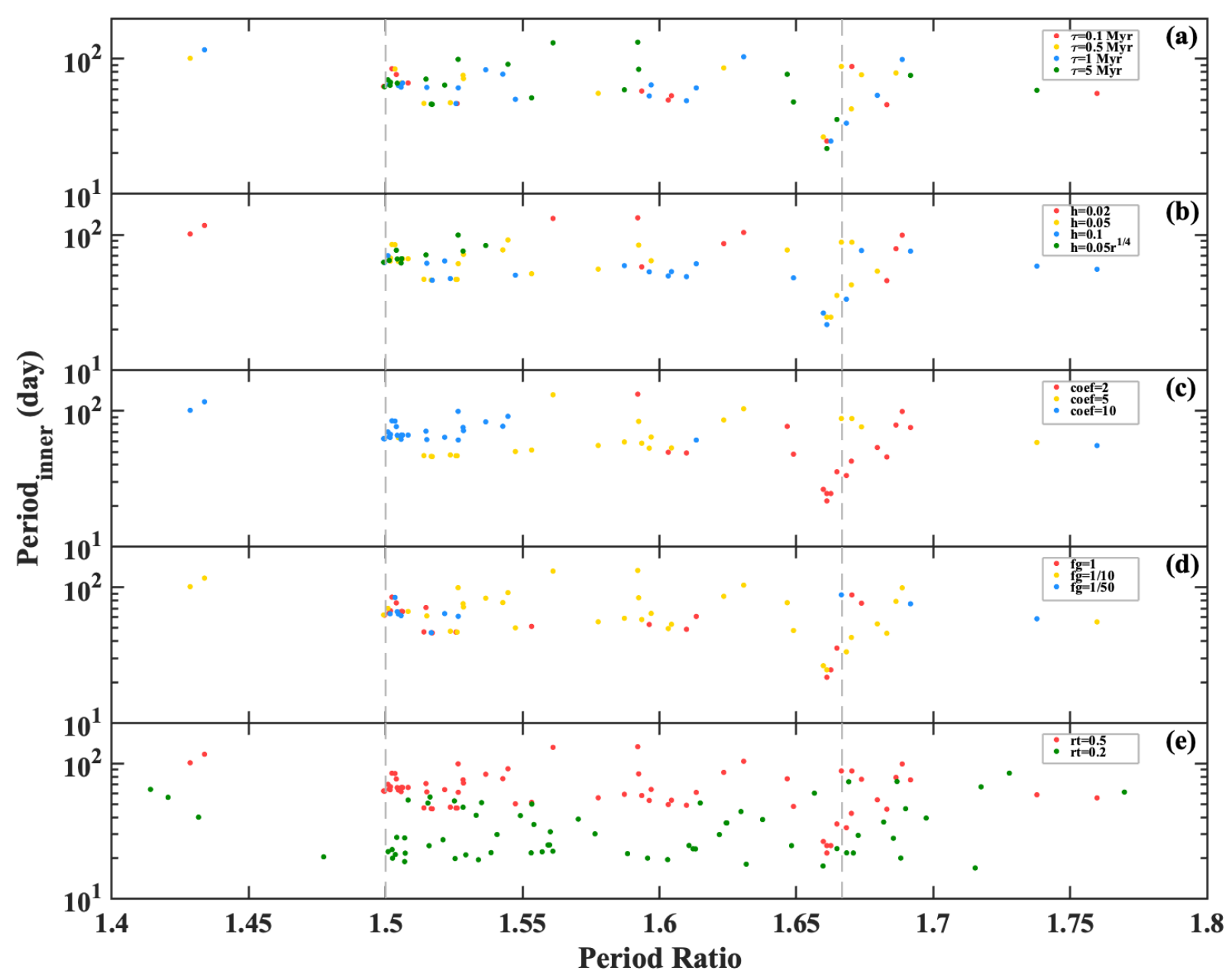

Figure 6. Distribution of planet pairs in the space of period ratio between 1.4 and 1.8 versus the period of inner planet. Panel (a)-(d) show the distribution of planet pairs changed with the depletion timescale of gas disk $\tau$, disk aspect ratio $h$, coefficient of $f_{n s c}$ coef, enhance factor of gas density $f_{g}$, and transition radius $r_{t}$, respectively.

or 5:3 MMRs through orbital migration with different migration speed. Specifically, a large number of simulations linked to 5:3 MMRs are produced with $h=0.05$ or $h=0.1$. For those systems harboring the innermost planets with a distance larger than $0.1 \mathrm{AU}$, the formation of near MMRs can be elucidated by the existence of proper depletion timescales of the gas disks. Due to the eccentricity damping induced by the gas disk, planet pairs can move out of the exact location of MMRs and be involved in near MMRs configurations. From our simulations, we show that the depletion of the gas disk can make the distribution of the period ratio comparable to the statistics of the observation.

2. With the depletion timescales larger than $1 \mathrm{Myr}$, we find that near MMRs configurations are easily to form. After planet pair is trapped into MMR, eccentricity damping is still strong enough to make planets move adequate distance to depart from exact MMR. Eccentricity damping plays a crucial role in leading to the deviation from the exact MMRs for planet pairs. Additionally, with the decrease of coef, 


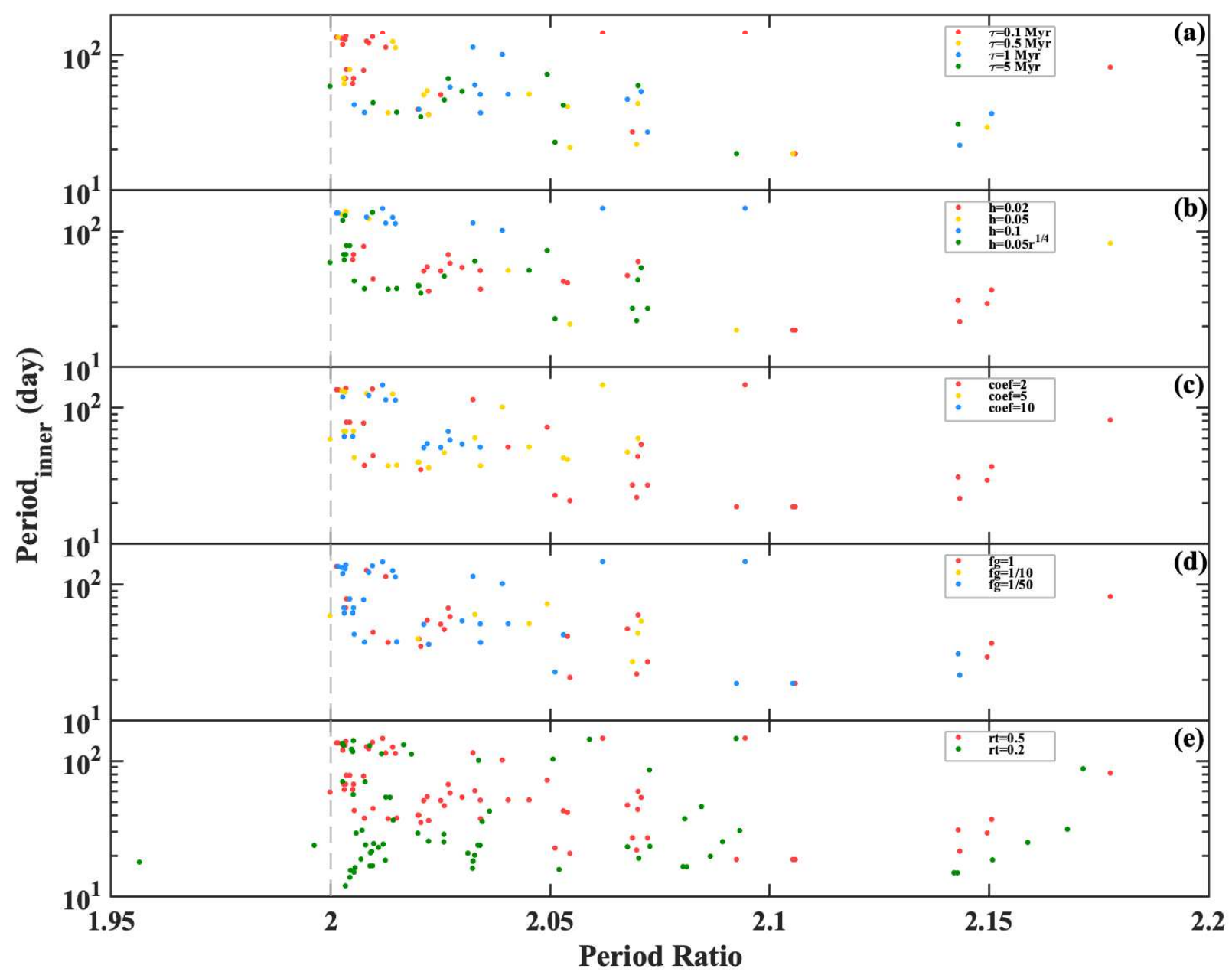

Figure 7. Distribution of planet pairs in the space of period ratio between 1.95 and 2.2 versus the period of inner planet. Panel (a)-(e) show the distribution of planet pairs changed with the depletion timescale of gas disk $\tau$, disk aspect ratio $h$, coefficient of $f_{n s c}$ coef , enhance factor of gas density $f_{g}$, and transition radius $r_{t}$, respectively.

which represents the strength of corotation torque, planet pairs can depart farther from the exact MMRs. Moreover, planet pairs have higher possibilities to escape from the configuration of MMRs with higher disk aspect ratio which means longer eccentricity damping timescales.

3. The final orbital periods of the innermost planets are directly related to the transition radius. If the innermost planets ranges in $[50,200]$ days, the transition radius of the disk is probably at about 0.5 $\mathrm{AU}$. In the case of the transition radius near $0.2 \mathrm{AU}$, the innermost planets can arrive at the orbital period 10 days. This suggests that the system with innermost planet closer to the central star holds stable planets at the later stage of star evolution process.

4. If the period ratios of planet pairs locate in the range of $[1.8,2.0]$, planets may be formed in the system with the gas disk of larger disk aspect ratio.

Tidal effect plays a significant part in giving rise to the departure of exact MMRs for the system with the extreme close-in innermost plan- 
ets the central star (Lee et al. 2013). From our work, we can conclude that after the planet pairs are captured into MMRs due to the migration process, planet pairs can depart from the exact MMRs locations because of the eccentricity damping caused by the gas disk for the systems with the innermost planets farther than $0.1 \mathrm{AU}$ in which the tidal effect of the star is not strong enough to affect the final configuration of the systems. Through our simulations, we can explain the distribution of the period ratio between the adjacent planets observed by the Kepler Mission. The timescale of the depletion of gas disk and disk aspect ratio are related to how far the planet pair departs from the exact MMRs and the location of the transition radius is connected with the final location of the innermost planets. The scenario can be also applied to explain the formation of planetary systems observed by TESS which may find a number of planet pairs in near MMRs (Quinn et al. 2019; Nielsen et al. 2020).

\section{ACKNOWLEDGMENTS}

This work is supported by the B-type Strategic Priority Program of the Chinese Academy of Sciences, Grant No. XDB41000000, National Natural Science Foundation of China (Grants No. 12033010, 11573073, 11633009, 11773081, 11761131008), CAS Interdisciplinary Innovation Team, Youth Innovation Promotion Association and the Foundation of Minor Planets of Purple Mountain Observatory.

\section{REFERENCES}

Aarseth S. J. 2003, Gravitational N-Body

Simulations, by Sverre J. Aarseth, pp. 430.

Cambridge, UK: Cambridge University Press, November 2003.

Antoniadou, K. \& Libert, A.-S., 2020, arXiv:2006.12895

Batalha N. M., Rowe J. F., Bryson S. T. et al. 2013, ApJS, 204, 24

Batygin K., \& Morbidelli A. 2013, AJ, 145, 1

Borucki, W. J., Koch, D., Basri, G. et al. 2010, Science, 327, 977

Chatterjee, S. \& Ford, E. B. 2015, ApJ, 803, 33

Delisle, J. -B., Laskar, J., \& Correia, A. C. M. 2014, A\& A, 566, A137

Dressing C. D. et al. 2017, AJ, 154, 207

Fabrycky D. C., Lissauer J. J., Ragozzine D., et al. 2014, ApJ, 146, 12

Gong Y.-X., Ji J.H., 2017, AJ, 154, 179

Gong Y.-X., \& Ji J.H., 2018, MNRAS, 478, 4565

Haisch K. E., Jr., Lada E. A., \& Lada C. J. 2001, ApJL, 553, L153

Hayashi C. 1981, Prog. Theor. Phys. Suppl., 70, 35

He, M. Y., Ford, E. B., Ragozzine, D., 2019, MNRAS, 490, 4575

Ida S., \& Lin D. N. C. 2004, ApJ, 604, 388

Ida S., \& Lin D. N. C. 2008, ApJ, 673, 487
Izidoro A., Ogihara M., Raymond S. N., Morbidelli A., Pierens A., Bitsch B., Cossou C., Hersant F., 2017, MNRAS, 470, 1750

Kley W., \& Nelson, R. P. 2012, ARA\& A, 50, 211

Koenigl A. 1991, ApJL, 370, L39

Kunimoto, M. \& Matthews, J. M. 2020, AJ, 159, 248

Lambrechts M., Morbidelli A., Jacobson S. A., Johansen A., Bitsch B., Izidoro A., Raymond S. N., 2019, A\&A, 627, A83

Lee M. H., \& Peale S. J. 2002, ApJ, 567, 596

Lee M. H., Fabrycky D., \& Lin D. N. C., 2013, ApJ, 774, 52L

Lissauer J. J., Ragozzine D., Fabrycky D. C., et al. 2011, ApJS, 197, 8

Lithwich Y. \& Wu Y. 2012, ApJ, 756, L11

Lin, D. N. C. \& Paploizou, J. 1979, MNRAS, 186, 799

Liu B. B., Zhang X. J., Lin D. N. C. 2015, ApJ, 798,62

Liu B. B., Ormel C. W., Lin D. N. C. 2017, A\& A, 601, A15

Liu B. B. \& Ji J. H. 2020, RAA, 20, 164

Mazeh T., et al. 2013, ApJS, 208, 16

Mills S. M. \& Fabrycky D. C. 2017, ApJL, 833, 6

Moriarty J. \& Ballard S. 2016, ApJ, 832, 34 
Morton T. D., Bryson, S. T., Coughlin, J. L. et al. 2016, ApJ, 822, 86

Murray, C. D., \& Dermott, S. F. 1999, Solar System Dynamics (Cambridge: Cambridge Univ. Press)

Nelson, R. P. \& Papaloizou, J. C. B., 2002, MNRAS, 333,26

Nielsen, L. D., Gandolfi, D., Armstrong, D. J. et al. 2020, MNRAS, 492, 5399

Ogihara M., Kokubo, E., Suzuki, T. K. et al., 2018, A\&A, 615, 63.

Owen J. E., \& Morton T. D. 2016, ApJL, 819, L10

Paardekooper S.-J., Baruteau C., Crida A., Kley W., 2010, MNRAS, 401, 1950

Paardekooper S.-J., Baruteau C., Kley W., 2011, MNRAS, 410, 293

Pan M. R., Wang S., Ji J. H., 2020, 496, 4688

Papaloizou, J. C. B. \& Szuszkiewicz, E. 2005, MNRAS, 363, 153

Papaloizou, J. C. B. \& Terquem, C. 2010, MNRAS, 405, 573

Petrovich C., Malhotra R., \& Tremaine S. 2013, ApJ, 770, 24.

Pierens, A. \& Nelson, R. P. 2008, A\& A, 482, 333

Quinn, S. N., Becker, J. C., Rodriguez, J. E., et al. 2019, AJ, 158, 177.

Ricker, G. R., Winn, J. N., Vanderspek, R., et al. 2015, Journal of Asstronomical Telescopes, Instruments, and Systems, 1, 014003
Rowe J. F., Bryson, S. T., Marcy, G. W. et al. 2014, ApJ, 784, 45

Steffen, J. H., Fabrycky, D. C., Agol, E. et al. 2013, MNRAS, 428, 1077

Steffen, J. H., \& Hwang, J. A. 2015, MNRAS, 448, 1956

Sun Z., Ji J. H., Wang S. et al. 2017, MNRAS, 467,619

Wang S., Ji J. H., \& Zhou J. L., 2012, ApJ, 753, 170

Wang S., \& Ji J. H., 2014, ApJ, 795, 85

Wang S., \& Ji J. H., 2017, AJ, 795, 85

Weiss L. M., \& Marcy G. W., 2014, ApJL, 783, L6

Williams, J. P., \& Cieza, L. A., 2017, ARA\&A, 49,67

Wu, D. H., Zhang, R. C., Zhou, J.-L. et al. 2019, MNRAS, 484, 1538

Xie, J. W. 2014, ApJS, 210, 25

Yang, J. Y, Xie, J. W, \& Zhou J.-L. 2020, AJ, 159,164

Zhang, X. 2020, RAA, 20, 7 